\title{
Glycogen synthase kinase 3B in bovine oocytes and granulosa cells: possible involvement in meiosis during in vitro maturation
}

\author{
Svetlana Uzbekova, Mohamad Salhab, Christine Perreau, Pascal Mermillod and Joëlle Dupont \\ Physiologie de la Reproduction et des Comportements, UMR6175 INRA, CNRS, Université de Tours, \\ Haras Nationaux, 37380 Nouzilly, France \\ Correspondence should be addressed to S Uzbekova; Email: svetlana.uzbekova@tours.inra.fr
}

\begin{abstract}
Glycogen synthase kinase 3 (GSK3) regulates cellular metabolism and cell cycle via different signalling pathways. In response to insulin and growth factors GSK3 is serine-phosphorylated and inactivated. We analysed GSK3B expression and activation in bovine cumulus cells (CC) and oocytes at different meiotic stages in vitro in parallel with MAP kinases ERK (MAPK3/MAPK1) and p38 (MAPK14). GSK3B localised to cytoplasm in granulosa cells and in oocytes throughout folliculogenesis. In mature metaphase-II (MII) oocytes, GSK3B was concentrated to the region of midzone between the oocyte and the first polar body, as well as active phospho-Thr Aurora A kinase (AURKA). During in vitro maturation (IVM), in oocytes, phospho-Ser'-GSK3B level increased as well as phospho-MAPK3/MAPK1, while phospho-MAPK14 decreased. In CC, phospho-MAPK14 increased upon germinal vesicle breakdown (GVBD)/metaphase-I (MI) and then decreased during transition to MII. Administration of inhibitors of GSK3 activity (lithium chloride or $2^{\prime} Z_{1} 3^{\prime}$ E -6-bromoindirubin-3'-oxime) rapidly increased phospho-Ser'-GSK3B, and led to transient decrease of phospho-

MAPK3/MAPK1 and to durable enhancing of phospho-MAPK14 in granulosa primary cell culture. GSK3 inhibitors during IVM diminished cumulus expansion and delayed meiotic progression. In cumulus, phospho-MAPK14 level was significantly higher in the presence of inhibitors, comparing with control, through the time of MI/MII transition. In oocytes, phospho-GSK3B was increased and phospho-MAPK3/MAPK1 was decreased before GVBD and oocytes were mainly arrested at MI. Therefore, GSK3B might regulate oocyte meiosis, notably MI/MII transition being the part of MAPK3/1 and MAPK14 pathways in oocytes and CC. GSK3B might be also involved in the local activation of AURKA that controls this transition.
\end{abstract}

Reproduction (2009) 138 235-246

\section{Introduction}

Glycogen synthase kinase 3 (GSK3) is a highly evolutionary conserved serine-threonine kinase, which was described first as a key enzyme in glycogen metabolism and was then found to be a multifunctional enzyme. GSK3 phosphorylates multiple substrates and is involved in regulation of protein synthesis, cell proliferation and differentiation, microtubule dynamics and apoptosis, being a part of $\mathrm{Wnt} / \beta$-catenin, NF- $\kappa \mathrm{B}$ and phosphatidylinositol 3-kinase $(\mathrm{PI} 3 \mathrm{~K}) /$ protein kinase $\mathrm{B}$ (PKB) signalling pathways (for review see (Ali et al. 2001)). GSK3 has two protein isoforms, $51 \mathrm{kDa}$ GSK3- $\alpha$ (GSK3A) and $46 \mathrm{kDa}$ GSK3- $\beta$ (GSK3B), which are encoded by different genes, highly similar in their kinase domains (Woodgett 1990). GSK3A and GSK3B are variably expressed in different tissues in humans and mice (Lau et al. 1999, Yao et al. 2002). In mammals, GSK3 is constitutively phosphorylated at Tyrosine ${ }^{279 / 216}$ residues and dephosphorylation on these sites is accompanied by kinase inactivation. In contrast, phosphorylation of serine ${ }^{21}$ and serine ${ }^{9}$ residues inactivates GSK3A and GSK3B respectively. This phosphorylation occurs in response to insulin, amino acids or polypeptide growth factors like epidermal growth factor (EGF) either by PKB (also named AKT) in PI3K/AKT pathway (Cohen et al. 1997), or by p70 S6 kinase in MTOR signalling or by MAPK-activated protein kinase 1 in classical MAPK cascade respectively (Cohen \& Frame 2001). Nucleotide polymorphisms in two components of the insulin signalling pathway, genes GSK3B and AKT2, were reported to be associated with polycystic ovary syndrome in women, characterised by different metabolic and reproductive disorders including hyperandrogenaemia and anovulation often associated with insulin resistance (Goodarzi et al. 2007, 2008). Cystic ovarian follicles are also a major cause of reproductive failure in high-lactating dairy cattle (Vanholder et al. 2006), which could also affect the oocyte quality (Bartolome et al. 2002). In vertebrates, GSK3 was shown to be involved in regulation of meiosis progression in frog oocytes 
(Fisher et al. 1999, Sarkissian et al. 2004) and in both male and female germ cells in mice (Guo et al. 2003, Wang et al. 2003, Liu et al. 2007). In mice, GSK3 is also involved in the regulation of chromatin segregation and cytokinesis in early embryo (Acevedo et al. 2007b). However, few data are currently available on GSK3 expression in ovary of human or large domestic animals and its involvement in oocyte maturation control. By antibody microarray analysis, GSK3A and GSK3B isoforms were recently detected in pig and bovine oocytes (Pelech et al. 2008).

In mammals, ovarian follicles contain the oocytes arrested at the first meiotic prophase. The process of oocyte nuclear maturation occurs in adult animals during the final growth of dominant follicles after $\mathrm{LH}$ surge and finished at ovulation. Meiosis restarts in oocyte with the condensation of chromosomes, germinal vesicle breakdown (GVBD), progression through metaphase-I (MI) and an arrest at metaphase-II stage (MII) before fertilisation. Fully grown bovine oocytes from follicles larger than $2 \mathrm{~mm}$ are capable to resume meiosis after in vitro maturation (IVM) and to develop up to the blastocyst stage after fertilisation (Sirard et al. 1988). Different signalling pathways are involved in the regulation of oocyte maturation in mammals. MAPK1 and MAPK3 (also known as ERK2 and ERK1 respectively) and maturation promoting factor (MPF), a complex of cyclin B1 and cell-cycle controller p34 kinase cyclindependent kinase 1 (CDK1) constituted the main signalling pathways of oocyte maturation control (see review (Liang et al. 2007)). We showed previously that several protein kinases are differentially activated in bovine oocytes during IVM: MAPK3/MAPK1, jun $\mathrm{N}$-terminal kinase (JNK), AKT, Aurora kinases (Vigneron et al. 2004a, 2004b, Uzbekova et al. 2008) and AMPactivated protein kinase (AMPK; Tosca et al. 2007). Another member of MAPK super-family, MAPK14 (alias p38) was expressed in porcine cumulus-oocyte complexes (COCs) and its activation varied during IVM (Villa-Diaz \& Miyano 2004). The involvement of different MAPKs in oocyte meiotic progression was reported also in mice, where levels of MAPK14, MAPK3/MAPK1 and JNK kinases' activities were shown to vary with the stage of oocyte maturation (Baatout et al. 2007). Members of insulin signalling cascade, including AKT and GSK3 were shown to play important roles in mice oocyte meiosis completion (Acevedo et al. 2007a). In addition, GSK3 was reported as the only kinase capable to phosphorylate Aurora-A in Xenopus oocytes (Sarkissian et al. 2004). We previously demonstrated that Aurora-A (AURKA) plays an important role in MI/MII transition in bovine oocytes (Uzbekova et al. 2008).

The aim of this study was to establish the spatiotemporal expression pattern of GSK3 in bovine oocytes and surrounding follicular cells during different stages of oocyte development and to investigate the potential role of GSK3 in oocyte maturation. To modulate the activity of GSK3 in our system, we chose a direct routinely used inhibitor, lithium chloride $\left(\mathrm{LiCl}_{2}\right)$ and a cell-permeable, highly potent, specific and reversible ATP-competitive inhibitor of GSK3, 2'Z, 3'E -6-bromoindirubin-3'-oxime (BIO). The mechanism of lithium double action on reducing GSK3 activity is well studied (Jope 2003) and $\mathrm{LiCl}_{2}$ inhibits GSK3, but not MPF activity (Meijer et al. 2004). GSK3-specific inhibitors as alsterpaullone and kenpaullone act in a similar way as $\mathrm{LiCl}_{2}$ in mouse embryonic cells (Acevedo et al. 2007b, Kaladchibachi et al. 2007). We first analysed GSK3 expression and phosphorylation patterns in oocytes and surrounding granulosa during folliculogenesis and throughout oocyte maturation by immunocytochemistry. MAPK3/1 and MAPK14 phosphorylation was analysed in parallel during IVM. Secondly, the dose and timecourse effects of GSK3 inhibitors on cell survival, progesterone secretion and activation of different MAPK pathways were explored on primary culture of granulosa cells from 3 to $6 \mathrm{~mm}$ bovine antral follicles. Finally, we analysed the effects of $\mathrm{LiCl}_{2}$ and $\mathrm{BIO}$ on meiosis progression and MAPK3/MAPK1 and MAPK14 phosphorylation in oocyte and cumulus cells (CC) during IVM.

\section{Results \\ GSK3 expression in bovine ovarian cells}

Human full-length GSK3A and GSK3B cDNA sequences (GenBank: NM_019884 and NM_002093 respectively) were used as queries to BLAST search in Bos taurus sequence collections accessible through GenBank. Using specific primers for B. taurus GSK3A and GSK3B orthologues, GSK3B cDNA fragment of expected size (171 bp) was detected by RT-PCR in oocyte and CC before and after IVM and also in brain, while GSK3A cDNA of expected size could be clearly detected only in the brain (Fig. 1A). Immunoblotting using the antibody to phospho-serine ${ }^{21 / 9}$ GSK3A/B, we only detected phospho-Ser ${ }^{9}$-GSK3B isoform in oocytes (Fig. 1B). This isoform was also the most abundant in $\mathrm{CC}$ and in brain. A $51 \mathrm{kDa}$ band that could correspond to phospho-Ser ${ }^{21}$ GSKA was detected in brain and faintly in CC. Thus, we considered only GSK3B for further analysis in oocyte and CC. Total GSK3B protein was detected at a similar level in immature and mature oocytes and correspondent CC. However, Ser ${ }^{9}$-phosphorylation of GSK3B was higher in mature compared with immature oocytes but not in CC. Data from western blots were confirmed by immunofluorescence (Fig. 1C, a and b). Inside COC, GSK3B was homogenously distributed in the cytoplasm and it was more abundant in CC compared with oocyte (Fig. 1C, a). In fully grown oocytes at germinal vesicle (GV) stage, GSK3B was significantly less phosphorylated at $\operatorname{Ser}^{9}$ than in oocytes 
A



$B$



C

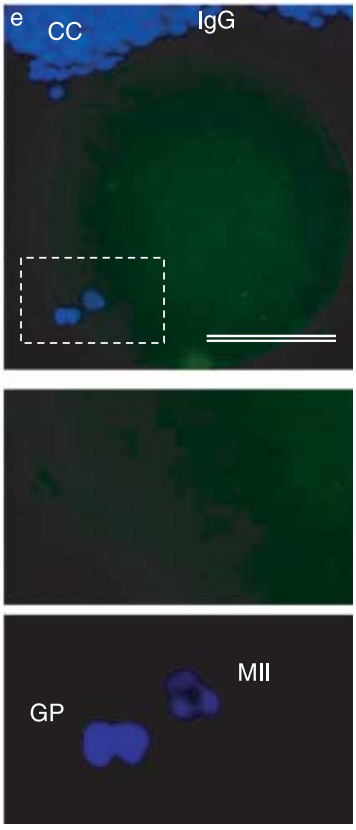

Figure 1 GSK3B mRNA and protein detection. (A) Detection by RT-PCR of GSK3B transcripts in cattle immature $(0 \mathrm{~h})$ and mature ( $24 \mathrm{~h}$ IVM) oocytes, cumulus cells (CC) and hypothalamic region of brain $(\mathrm{Br})$. Control $\beta$-actin (AKTB) transcripts were detected in the same cDNA samples. (B) Total GSK3B protein $(46 \mathrm{kDa})$ and phospho-Ser ${ }^{21 / 9}$ GSK3A/B detection by western blot. Total proteins were extracted from oocytes and cumulus cells before or after $24 \mathrm{~h}$ IVM and from brain biopsy, and then subjected to SDSPAGE and immunoblot. In total, 40 immature and mature oocytes and cumulus cells from 10 COCs were loaded per lane. (C) Immunofluorescence of GSK3B in the cumulus-oocyte complex (green) showed that GSK3B was less detected in oocyte as compared with cumulus cells, CC (a). Phospho-Ser ${ }^{9}$ GSK3B was less detected in germinal vesicle immature oocytes (GV) compared with oocytes that over-passed the GVBD as demonstrated by simultaneous phospho-Ser ${ }^{9}$ GSK3B (green) and Lamin A/C nuclear membrane (red) staining (b). In metaphase-II oocytes, GSK3B (c) and phospho-Thr AURKA (d) localised to a contractile ring between the ooplasm and polar body (encompassed by white arrows). White-dotted line rectangles encompass the regions, which are magnified below. Chromatin is blue-coloured by DAPI. Bars: $50 \mu \mathrm{m}$. already passed through GVBD after IVM (Fig. 1C, b). In mature oocytes, GSK3B was particularly concentrated closely to a polar body/oocyte border, that might be a contractile ring, bundling microtubule-based structure (Fig. 1C, c). The active form of Aurora A kinase, phospho-Thr AURKA, was also localised to this structure (Fig. 1C, d), as we already reported (Uzbekova et al. 2008). No significant fluorescence was observed in oocyte and CC, if rabbit IgG replaced the first antibodies.

We then analysed the localisation of GSK3B and its serine-phosphorylated form throughout folliculogenesis by $3,3^{\prime}$-diaminobenzidine (DAB)-immunostaining on the paraffin-embedded sections of bovine ovaries, containing the follicles of different stages from primordial to antral (Fig. 2). GSK3B was not detected in interstitial tissue, but was clearly immunostained in primordial and primary follicles both in oocytes and in a monolayer of surrounding granulosa cells (Fig. 2A). In secondary and antral follicles, GSK3B was detected in oocytes, in granulosa and in theca cell layers (Fig. 2D and G). In large antral follicles, GSK3B was detected in CC and much less in oocyte cytoplasm (Fig. 2J). No significant immunoreactivity was detected in control adjacent sections stained with rabbit IgG (Fig. 2B, E, H and $\mathrm{K}$ ). In secondary preantral and antral follicles, phospho-Ser ${ }^{9}$ GSK3B was detected in granulosa cells and in CC. Nevertheless, oocytes also showed a slight immunoreactivity (Fig. 2C, F, I and L). 





Figure 2 Localisation of GSK3B in bovine ovary. Analysis of total GSK3B protein expression (A, D, $G$ and $J$ ) and its Ser ${ }^{9}$-phosphorylated form, pSer-GSK3B (C, F, I and L) during folliculogenesis was performed by DAB-immunoperoxidase staining on paraffin-embedded ovary sections (immuno-specific staining is brown and cell nuclei are violet). GSK3B was detected in oocytes and surrounded follicular cells in different follicles: primary $(A)$, secondary pre-antral (D), tertiary small antral (G) and in COCs from large antral follicles (J). Serine-phosphorylated GSK3B was detected mainly in granulosa and cumulus cells $(\mathrm{C}, \mathrm{F}, \mathrm{I}$ and $\mathrm{L})$ and more faintly in oocytes. Control immunostaining was performed by using rabbit $\lg \mathrm{G}$ instead of primary antibodies on adjacent sections (B, E, H and K). Cumulus-oocyte complex (COC), oocyte (Oo), cumulus cells (CC), antrum $(\mathrm{An})$, granulosa $(\mathrm{Gr})$, theca cells $(\mathrm{Th})$ and interstitial tissue (Int) are designated. Bars: $50 \mu \mathrm{m}$.

\section{Phosphorylation of GSK3B, MAPK3/MAPK1 and MAPK14 in oocytes and CC during IVM}

Once extracted from the antral follicles and transferred into IVM culture medium for $24 \mathrm{~h}$, bovine oocytes inside of the COC passed through the sequential stages of nuclear maturation and finally stopped at MII stage. In our conditions, the recovered oocytes were in meiotic prophase and retained the intact GV for at least $6 \mathrm{~h}$. Initially GVBD occurred after $\sim 6-7 \mathrm{~h}$ of IVM and the Ml stage was achieved at 10-12 h of IVM. At 22-24 h, most of the oocytes were mature and arrested at MII. We analysed GSK3B, MAPK3/MAPK1 and MAPK expression by immunoblot in both oocyte and CC at different time points of IVM (Fig. 3). In oocytes, progressive GSK3B phosphorylation at $\mathrm{Ser}^{9}$ occurred throughout IVM and reached a maximum at MII stage, being more than 12 times increased as compared with immature oocytes (Fig. 3, left). A similar evolution was observed for phospho-MAPK3/MAPK1 level, which also significantly increased. In contrast, phosphoMAPK14 decreased drastically in mature oocytes as compared with immature ones.
In CC, GSK3B was already Ser-phosphorylated before IVM, phospho-GSK3B increased 1.5 times at the beginning of the culture and then maintained slightly variable phosphorylation level (Fig. 3, right). We previously reported a similar pattern of MAPK3/MAPK1 phosphorylation in CC during IVM (Tosca et al. 2007), i.e. slight increase from the beginning of maturation and the maintenance at that level. In contrast, phosphoMAPK14 level increased more than three times in CC between 1 and $10 \mathrm{~h}$ of IVM and then dropped to the initial level after $24 \mathrm{~h}$ of culturing.

Thus, we showed that GSK3B, MAPK14 and MAPK3/ MAPK1 were present in oocyte and surrounding CC and their phosphorylation levels were modulated during IVM.

\section{Effect of GSK3 inhibitors on bovine oocyte nuclear maturation in vitro}

To look for a potential function of GSK3B in oocyte, we first used $\mathrm{LiCl}_{2}$ as a direct inhibitor of GSK3 kinase activity. Final concentration of $20 \mathrm{mM} \mathrm{LiCl}_{2}$ previously shown to be non-toxic for mouse oocytes and embryos 



Figure 3 Immunoblot detection of GSK3B, MAPK3/1 and MAPK14 total proteins and their phosphorylation forms in oocytes and cumulus cells before IVM $(0 \mathrm{~h})$ or after 3, 6, 10 and $22 \mathrm{~h}$ of IVM. Denuded oocytes (30 oocytes per lane) or CC from ten COCs were subjected to immunoblot by using successively antibodies, detecting phosphorylated or total proteins on the same membrane. Representative blots from at least three experiments are shown. Signals were quantified and ratios of phosphoprotein kinase/total protein kinase are represented as histograms (mean ratio \pm s.E.M.; the level in immature oocytes was considered as one). Different letters designated a significant difference $(P<0.05)$.

(Acevedo et al. 2007b), was used in these studies. The addition of $20 \mathrm{mM}$ of $\mathrm{LiCl}_{2}$ to bovine COC during IVM led to reduced expansion of cumulus after $24 \mathrm{~h}$ of IVM as compared with control (Fig. 4A, left column). All lithium-treated oocytes accomplished GVBD, as was confirmed by lamin $\mathrm{A} / \mathrm{C}$ immunostaining (not shown), but were then arrested in MI-like stage (Fig. 4A). GSK3B distribution after IVM differed between the oocytes matured with or without $\mathrm{LiCl}_{2}$ : in control matured oocytes at MII stage, GSK3B was particularly concentrated in the region of polar body (which could be the midbody or contractile ring), while no particular concentration was observed in Ml-arrested lithiumtreated oocytes. Chromosome alignment was disturbed in Ml figures observed in lithium-treated oocytes and the spindle was asymmetric (Fig. 4B).

In control IVM, $89.7 \%$ attained MII and only $10.3 \%$ did not progress beyond $\mathrm{MI}$, while in the lithium-treated group $85.9 \%$ of oocytes were blocked in the first metaphase (Fig. 4C). When $\mathrm{BIO}$ inhibitor was added, a dose-dependent decrease of oocyte nuclear maturation level was observed, being reduced more than twice at $50 \mu \mathrm{M}$ concentration. Moreover, the cumulus expansion was also significantly reduced at a concentration higher than $10 \mu \mathrm{M}$ (data not shown).

Thus, we showed that addition of GSK3 inhibitors $\mathrm{LiCl}_{2}$ or BIO during IVM reduced cumulus expansion and delayed oocyte maturation by blocking $\mathrm{MI} / \mathrm{MII}$ transition in bovine oocyte.

\section{Effect of $\mathrm{LiCl}_{2}$ on cell survival and progesterone secretion by granulosa cells and COC in vitro}

In order to explain the possible reasons of meiotic arrest of oocytes in COC cultured with $\mathrm{LiCl}_{2}$, we first looked for the dose-dependent effect of $\mathrm{LiCl}_{2}$ on cell survival and proliferation level on primary culture of granulosa cells in the presence of increasing concentrations of $\mathrm{LiCl}_{2}$. At $20 \mathrm{mM}$ no impact was observed in granulosa cells proliferation level as compared with untreated cells (Fig. 5A). Nevertheless, significant decrease in progesterone secretion by granulosa cells was observed from a concentration of $10 \mathrm{mM}$ and higher. At $20 \mathrm{mM}$, progesterone secretion was three times less than in control (Fig. 5B). This decrease was observed both in basal and FSH- or insulin-like growth factor-I (IGF-I)-supplemented medium (Fig. 5C). COC also secreted progesterone during IVM, and its level increased mostly between 10 and $24 \mathrm{~h}$ of maturation (Fig. 5D, line-curve graph). Similar to granulosa cells, in the presence of lithium COC secreted two-fold less progesterone than in control IVM conditions.

\section{Effect of GSK3 inhibitors on the phosphorylation of GSK3B, MAPK3/MAPK1 and MAPK14 in bovine granulosa cells, $C C$ and oocyte in vitro}

After $\mathrm{LiCl}_{2}$ addition in granulosa cell culture we observed very rapid (already $1 \mathrm{~min}$ after lithium administration) increase of phospho-Ser ${ }^{9}$-GSK3B level (Fig. 6A). In order to test what signalling pathways have been affected by lithium and could be involved in oocyte meiotic arrest, we then quantified the phosphorylation of MAPK3/ MAPK1, AKT, AMPK and MAPK14. Neither AMPK nor AKT phosphorylation were affected by $\mathrm{LiCl}_{2}$ both in granulosa cells during the first $60 \mathrm{~min}$ of treatment and in CC after $24 \mathrm{~h}$ IVM (data not shown). Phospho-MAPK3/ MAPK1 level significantly decreased in granulosa cells 10 min after lithium administration and then remained stable during 60 min of observation. In contrast, phosphoMAPK14 level significantly and constantly increased at the same time points. In the presence of BIO inhibitor, phospho-Ser ${ }^{9}$-GSK3B level was progressively increasing during $60 \mathrm{~min}$. At this time point, dose-dependent decrease of phospho-MAPK3/1 and increase of phospho-MAPK14 was also observed (Fig. 6B).

In CC from lithium-treated or control COC, no differences in phospho-GSK3B and phospho-MAPK3/1 levels were observed at 1, 3, 6, 9, 18 or $24 \mathrm{~h}$ of IVM (data not shown). However, in the presence of either $\mathrm{LiCl}_{2}$ or $\mathrm{BIO}$, phospho-MAPK14 level in CC was stably higher than in control during IVM (Fig. 7A). In oocytes at $6 \mathrm{~h}$ of IVM, we observed two-time higher level of phosphoGSK3B and eight-time lower level of phospho-MAPK3/1 in lithium-treated group (Fig. 7B). These effects were already visible at $3 \mathrm{~h}$ of IVM; however, at the end of maturation (at $24 \mathrm{~h}$ ) no more differences were observed (data not shown). 
A




MergeGSK3B + DAPI


B

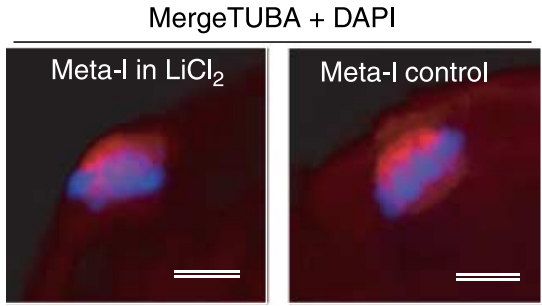

C


Figure 4 Effect of $\mathrm{LiCl}_{2}$ and GSK3 inhibitor $\mathrm{BIO}$ on maturation of bovine oocytes in vitro. (A) COCs expansion (first column, bar $500 \mu \mathrm{m}$ ), stage of oocyte chromatin (blue DAPI-staining) and GSK3B immunofluorescence (green) were analysed before $(0 \mathrm{~h})$ and after $24 \mathrm{~h}$ IVM (second column, bars $50 \mu \mathrm{m}$ ). White-dotted line rectangles encompassed regions that are magnified on the right pictures. Region of particular GSK3B concentration near the polar body (PB) in control mature oocyte is encompassed by white arrow. (B) Spindle detection by TUBA immunofluorescence (red) in metaphase-I control and $\mathrm{LiCl}_{2}-$ treated oocytes; chromosomes (blue) are misaligned in the presence of lithium. Bars $10 \mu \mathrm{m}$. (C) Histogram representing the percentage of mature oocytes after $24 \mathrm{~h}$ of IVM in control or in GSK3 inhibitor-treated oocytes. $20 \mathrm{mM} \mathrm{LiCl}$ (right graph) or different concentration of BIO (left graph) was used. Different letters designated a significant difference $(P<0.05)$.
Taking together, GSK3 inhibitors $\mathrm{LiCl}_{2}$ and $\mathrm{BIO}$ increased phospho-Ser ${ }^{9}$-GSK3B and phospho-MAPK14 levels, decreased MAPK3/MAPK1 phosphorylation in bovine granulosa and CC in vitro, and delayed oocyte maturation by blocking transition from MI to MII (Fig. 8).

\section{Discussion}

\section{GSK3B expression in bovine oocytes and surrounding follicular cells}

We showed that GSK3B is present in bovine oocytes and in surrounding follicular cells throughout meiosis from primary follicles up to ovulation stage. Between two GSK3 isoforms, GSK3B was the most abundant in bovine oocytes and in CC. Effectively, the expression of GSK3A and GSK3B varied in different tissues of mammals with a poor correlation between mRNA and protein levels, reflecting the differential regulation of transcription and translation for these highly similar kinases (Woodgett 1990, Lau et al. 1999). GSK3A mRNA was not detected in mice oocytes and embryos, whereas both GSK3A and GSK3B proteins were evidenced and differentially phosphorylated (Wang et al. 2003, Acevedo et al. 2007 b). GSK3 is involved in the regulation of transcription by phosphorylation or destabilisation of transcriptional factors, maintaining them in an inactive state under basal conditions (Frame \& Cohen 2001). Transcriptional factors c-Myc, c-Jun and CREB, known substrates of GSK3, are also expressed in mammalian oocytes (Li et al. 1994, Vigneault et al. 2004, Baatout et al. 2007). In bovine follicles, GSK3B is expressed continuously during oogenesis and meiosis progression, thus transcription activity might be partially regulated via GSK3B pathway in bovine oocyte. GSK3 is also known 

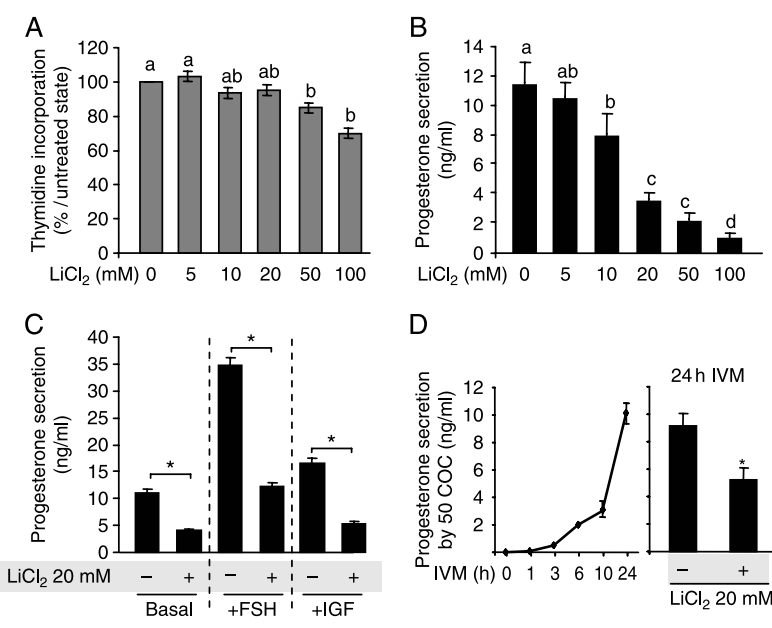

$\mathrm{D}$



Figure 5 Effect of different concentrations of $\mathrm{LiCl}_{2}$ on (A) thymidine incorporation and on (B) progesterone secretion by primary culture of bovine granulosa cells in vitro. (C) Effect of $20 \mathrm{mM} \mathrm{LiCl}_{2}$ on progesterone secretion by granulosa cells in basal culture medium and in response to FSH or IGF-1. (D) Time course of progesterone secretion by COCs during IVM (curve line) and final progesterone concentrations at $24 \mathrm{~h} \mathrm{IVM} \mathrm{in}$ control and $\mathrm{LiCl}_{2}$ supplemented medium (histogram). Different letters and asterisks designated a significant difference $(P<0.05)$.

to regulate protein synthesis: when GSK3B is Serphosphorylated in response to insulin, eukaryotic protein synthesis initiation factor $2 \mathrm{~B}$ is activated and contributes to an increased rate of protein synthesis (Welsh et al. 1998). In bovine oocytes, protein neosynthesis is indispensable for meiosis resumption and progression. A significant increase in overall protein synthesis has been reported in cattle oocytes at 6-10 h after beginning of IVM (Tomek et al. 2002), and numerous proteins were translated de novo (Coenen et al. 2004). Coincidently, GSK3B was shown to be progressively inactivated, i.e. phosphorylated at Serine ${ }^{9}$ during IVM and was localised in the cytoplasm. Therefore, GSK3B might also participate in the regulation of protein synthesis in oocytes. Recent data showed that in mouse oocyte, soluble kit ligand $(\mathrm{KL})$, a protein expressed in granulosa cells and indispensable for follicular and oocyte growth, could inactivate GSK3 via KL-Kit-PI3K-AKT pathway (Liu et al. 2007). Expression of $K L$ was reported in bovine ovarian surface epithelium, stroma and granulosa cells (Parrott et al. 2000). We detected GSK3B in granulosa cells from primary follicular stage and through further follicular growth. Thus, GSK3B may participate in the regulation of early follicular development and oocyte growth.

We detected GSK3B into cytoplasm but not in the nucleus of immature growing and fully grown oocytes, like in mice (Wang et al. 2003). Ser ${ }^{9}$-phosphorylated inactive GSK3B was detected in mice MII oocytes (Acevedo et al. 2007b), and we found that this form was more abundant in mature bovine oocytes compared with immature ones. Similarly, in Xenopus, GSK3B was active in GV oocytes and inactivated during maturation in response to progesterone (Fisher et al. 1999).

In bovine mature oocytes, GSK3B was particularly concentrated to a region separating the polar body from oocyte, what could be a part of a midbody or contractile ring. No particular concentration of $\mathrm{pSer}^{9}$-GSK3B was detected at that site; therefore, GSK3B could be active at the contractile ring/midbody. Aurora kinases AURKB, AURKC and active Thr-phosphorylated form of AURKA were also detected at this particular site in mature oocytes (Uzbekova et al. 2008). In Xenopus oocytes GSK3 was reported to phosphorylate Aurora-A at Serine ${ }^{349}$, inhibiting its kinase activity and in turn stimulating progesterone-triggered oocyte maturation (Sarkissian et al. 2004). Indeed, this phosphorylation of Aurora-A at Serine $^{349}$ was found to be indispensable for meiosis

\section{A}


B




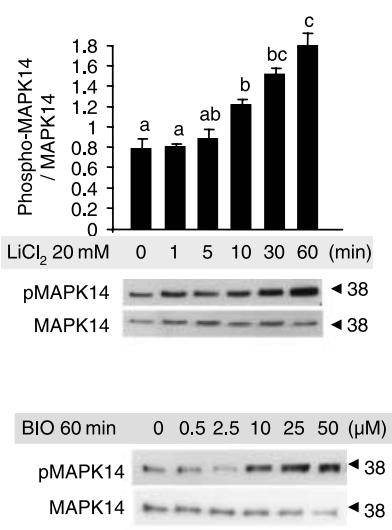

Figure 6 (A) Time-course effects of $\mathrm{LiCl}_{2}$ on phosphorylation of GSK3B, MAPK3/MAPK1 and MAPK14 in bovine granulosa cells in vitro. Different concentrations of $\mathrm{LiCl}_{2}$ were added to primary granulosa cells (48 h culturing) and cells were lysed after 1, 5, 10, 30 or 60 min after beginning of treatment. Quantified values from western blot signals (three replicates) were presented on histograms as a ratio phosphorylated protein kinase/total protein kinase. Different letters designated a significant difference $(P<0.05)$. (B) Time-course effect of $10 \mu \mathrm{M} B \mathrm{O}$ on GSK3B phosphorylation (left picture) and dose-dependent effect of BIO on MAPK3/MAPK1 and MAPK14 phosphorylation in granulosa cells in vitro. Extracted proteins were separated by SDS-PAGE and immunoblots with different antibodies were performed on the same samples. Representative western blots from two independent experiments are shown. 
A


B

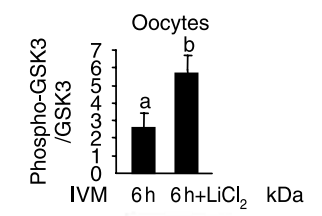

pSer-GSK3B $\longrightarrow 46$

GSK-3B $\longrightarrow 46$

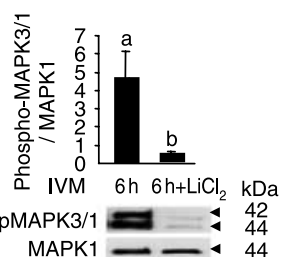

Figure 7 (A) Effect of $20 \mathrm{mM} \mathrm{LiCl}_{2}$ or $10 \mu \mathrm{M} \mathrm{BIO}$ on MAPK14 phosphorylation in cumulus cells during IVM. Proteins were extracted from 10 COCs after 6, 9, 18 and $24 \mathrm{~h}$ of IVM culture and analysed by western blot. (B) Effects of $20 \mathrm{mM} \mathrm{LiCl}_{2}$ on phosphorylation of GSK3B and MAPK3/MAPK1 in oocytes after $6 \mathrm{~h}$ IVM. Different letters designated a significant difference $(P<0.05)$.

progression in Xenopus oocytes (Pascreau et al. 2008). In human HeLa cells, it was recently shown that GSK3B finely regulated synthesis and activity of AURKA through binding and phosphorylation of Aurora-A-interacting protein (AIP) during mitosis (Fumoto et al. 2008). At the same time, GSK3B, AIP and AURKA were co-localised at the spindle poles, and when AIP was depleted, AURKA remained permanently active and caused a pro-metaphase arrest (Fumoto et al. 2008). We detected active phospho-Thr AURKA in cytoplasm of bovine immature oocytes and at the contractile ring of midbody in mature MIl-arrested oocytes (Uzbekova et al. 2008). Similar pattern for GSK3B was observed in the present work. AURKA inactivation induced aberrant chromosome distribution and inhibition of polar body extrusion in bovine and mice oocytes (Swain et al. 2008, Uzbekova et al. 2008). GSK3 inhibition also led to chromosome misalignment and thus, meiosis could not be completed (Wang et al. 2003, and present study). Thus, GSK3B might be involved in the regulation of AURKA activity in mammalian oocyte, although mechanisms of GSK3B effects on AURKA remain to be clarified.

During IVM, the level of phosphoSer ${ }^{9}$-GSK3B in CC increased rapidly in response to components like insulin and EGF contained in IVM culture medium. In oocytes, GSK3B Ser ${ }^{9}$-phosphorylation significantly increased from GVBD stage, in parallel with the increasing of progesterone secretion after $10 \mathrm{~h}$ of IVM, which is the period of MI/MII transition. Variations of GSK3B activity in oocytes and progesterone secretion by correspondent COC were negatively correlated during lithium treatment: in the presence of $\mathrm{LiCl}_{2}$ progesterone synthesis decreased; however, GSK3B activity was diminished. In human endometrial-derived epithelial cell line, progesterone induced a dose-dependent increase in

phospho-GSK3B, which could be blocked by competitive progesterone receptor antagonist RU486 (Salameh et al. 2006). This suggests that progesterone might regulate the expression of GSK3. In Xenopus oocytes, progesterone and insulin stimulated egg's maturation by inhibiting GSK-3 kinase activity (Sarkissian et al. 2004). Therefore, intra-follicular pools of GSK3B in bovine might also be regulated by progesterone.

\section{Possible mechanism of meiotic arrest mediated by GSK3 inhibitors in bovine COC during IVM}

When IVM was performed in the presence of lithium or $\mathrm{BIO}$, oocytes could not progress beyond $\mathrm{MI}$ stage, which occurred after 9-12 $\mathrm{h}$ as expected. Chromosomes were misaligned in lithium-treated MI-like oocytes although spindle was present. In mice early embryos treated with lithium or with another GSK3 inhibitor alsterpaullone, chromatin segregation abnormalities were also observed during the first cleavages (Acevedo et al. 2007b). Recently, GSK3B has been found to be involved in the stabilisation of meiotic spindle, and it was localised to spindle poles and to kinetochore region in mature mice oocytes (Baluch \& Capco 2008). MAPK14 is also considered as a component of the spindle assembly checkpoint in cell cycle. Addition of activated recombinant p38 to Xenopus cell-free extracts or in early embryo caused arrest in M phase (Takenaka et al. 1998).



Figure 8 Hypothetical schema of involvement of GSK3B in oocyte transition from metaphase-I to metaphase-II concluded from the experiences with GSK3 inhibitors. Phospho-Ser ${ }^{9}$-GSK3B, phosphoMAPK3/1 and phospho-MAPK14 are present in COC at metaphase-I. During the period of 10-24 h of IVM, cumulus is largely expanded, progesterone secretion increased and level of phospho-MAPK14 diminished in CC and in oocyte. In oocyte, remains of active GSK3B might participate in phosphorylation of AURKA, which is involved in regulation of chromosome segregation and MI/MII transition. Oocyte thus, reached correctly metaphase-II stage. In the presence of GSK3 inhibitors, cumulus was not expanded and progesterone secretion diminished. GSK3 inhibitors led to the additional Ser ${ }^{9}$-phosphorylation of GSK3B, decreased phospho-MAPK3/1 level and maintained phosphoMAPK14 at a higher level, which could hamper the normal meiosis progression. When inhibitors inactivated GSK3B, local phosphorylation of AURKA might not occur, spindle was not correctly formed and oocyte was blocked at metaphase-I. 
In our experiments, we observed an increase of phospho-GSK3B and phospho-MAPK14 and a decrease of MAPK3/MAPK1 phosphorylation in granulosa cells in response to lithium or BIO. During IVM, these effects were associated with the decrease of progesterone secretion and with the arrest of oocytes at MI-like stage as resumed in Fig. 8. Both $\mathrm{LiCl}_{2}$ and $\mathrm{BIO}$ induced a stable phosphorylation of MAPK14 in bovine CC during IVM. During IVM, MAPK14 was progressively phosphorylated in bovine $\mathrm{CC}$ with a maximum at $6-10 \mathrm{~h}$, corresponding to GVDB-MI transition. Then, phospho-MAPK14 level in cumulus decreased drastically during MI/MII transition. During this period (10-24 h of IVM), cumulus expanded and gap junctions closed between CC and oocyte. Specific inhibition of p38/MAPK14 phosphorylation by SB203580 during IVM led to a drastic decrease of cumulus expansion in porcine $\mathrm{COC}$ and to the arrest of oocytes at GV stage (Villa-Diaz \& Miyano 2004). Lithium and $\mathrm{BIO}$ also inhibited the expansion in bovine COC during IVM. We directly showed by immunoblot that MAPK14 was phosphorylated in immature oocytes, whereas phosphorylation mostly disappeared after IVM. Similarly, in sea star immature oocytes, MAPK14 orthologue was Tyr-phosphorylated and dephosphorylation occurred during maturation (Morrison et al. 2000). In mice, p38 $\gamma$ isoform was shown to be phosphorylated since GVBD (Perdiguero et al. 2003). In pig oocytes, after observation of ATF-2, a p38 substrate, it was supposed that p38 activation occurred after GVBD (Villa-Diaz \& Miyano 2004). In fact, different isoforms of p38 MAPK exist and their activation is tissue specific (Zarubin \& Han 2005). Thus, MAPK14 might participate in meiosis progression by modulating its activity.

$\mathrm{LiCl}_{2}$ was shown to mimic insulin induced stimulation of glucose transport, and this effect was associated with a significant enhancement of GSK3B and MAPK14 phosphorylation in muscle adipocytes (MacAulay et al. 2003). Lithium also enhanced basal and insulinstimulated MAPK14 phosphorylation in insulin-sensitive rat skeletal muscle (Harrell et al. 2007). Our data demonstrated, that in the presence of lithium or $\mathrm{BIO}$, MAPK14 phosphorylation in COC was maintained at a higher level at the time of MI/MII transition compared with control IVM. We hypothesised that MAPK14 could be involved in the regulation of meiosis progression and is co-regulated with GSK3B. Recently, direct phosphorylation of GSK3B at serine ${ }^{389}$ by p38/MAPK14 has been reported, and this is a tissue-specific alternative pathway for GSK3B inactivation in mammalian cells (Thornton et al. 2008). In our experiments, inactivation of GSK3B by lithium correlated in oocytes with a transient decrease of MAPK3/MAPK1 activity preceding GVBD (at $3-6 \mathrm{~h}$ of IVM). Nevertheless, this might explain the delay in meiosis progression and the block of MI/MII transition. When MAPK3/MAPK1 activity was blocked by specific MEK1/2 inhibitor U0126 from the beginning of IVM, bovine oocytes progress through
GVBD, but could not reach the correct MI stage (Tosca et al. 2007); the same effect was reported in mice (Lee et al. 2007). In contrast, when mouse oocytes were treated by U0126 after GVBD, although the phosphorylation of MAPK3/MAPK1 was drastically reduced, the polar body extrusion was normal, but the organisation of metaphase plate and chromosome segregation were affected (Lee et al. 2007). Thus, in bovine oocytes, $\mathrm{LiCl}_{2}$ led to the inhibition of MAPK3/MAPK1 activation prior to GVBD, which in turn contributed to the arrest at first meiotic division.

In conclusion, spatio-temporal pattern of GSK3B expression and activation in bovine oocytes and surrounding granulosa cells together with a functional analysis of GSK3B by modification of its activity by using specific GSK3 inhibitors, pointed to several possible functions of this kinase in oocyte meiosis. First, GSK3B is probably involved in follicle and oocyte growth during folliculogenesis. Second, during the first meiotic division, GSK3B could act as a regulator of chromosome segregation and correct Ml/MII transition by interacting with AURKA. As in other tissues, GSK3B takes part in different signalling pathways; nevertheless in COC, GSK3B-MAPK14 interactions seem to contribute to cumulus expansion and transition of oocyte from $\mathrm{MI}$ to MII.

\section{Materials and Methods}

\section{Ethics}

All procedures were approved by the Agricultural and Scientific Research Government Committees in accordance with the guidelines for Care and Use of Agricultural Animals in Agricultural Research and Teaching (approval A37801).

\section{Materials}

All substances for in vitro culture of bovine oocytes and granulosa cells and molecular grade $\mathrm{LiCl}_{2}\left(8 \mathrm{M} \mathrm{LiCl}_{2}\right)$ were purchased from Sigma.

$\mathrm{BIO}$ was from Calbiochem (VWR, Meuden, France). Purified ovine FSH-20 (oFSH; lot no.AFP-7028D, $4453 \mathrm{IU} / \mathrm{mg}, \mathrm{FSH}$ activity $=175$ times activity of (oFSH-S1)) used for culture treatment was a gift from NIDDK, National Hormone Pituitary Program, Bethesda, MD, USA.

\section{Samples collection}

Cattle ovaries were collected from a slaughterhouse. COCs were aspirated from 3 to $6 \mathrm{~mm}$ antral follicles. COC with more than three layers of compact CC surrounding the oocyte were selected and washed several times in TCM199/HEPES medium supplemented with $50 \mathrm{mg} / \mathrm{l}$ of gentamycin. Groups of 50 COC were subjected to IVM in $500 \mu \mathrm{l}$ of TCM199 serum-free medium supplemented with EGF (10 ng/ml), IGF-1 (19 ng/ml), fibroblast growth factor $(2.2 \mathrm{ng} / \mathrm{ml})$, hCG $(5 \mathrm{Ul} / \mathrm{ml})$, pregnant mares serum gonadotrophin $(10 \mathrm{Ul} / \mathrm{ml})$, insulin $(5 \mu \mathrm{g} / \mathrm{ml})$, 
transferrin $(5 \mu \mathrm{g} / \mathrm{ml})$, selenium $(5 \mathrm{ng} / \mathrm{ml})$, L-cystein $(90 \mu \mathrm{g} / \mathrm{ml})$, $\beta$-mercaptoethanol $(0.1 \mathrm{mM})$, ascorbic acid $(75 \mu \mathrm{g} / \mathrm{ml})$, glycine $(720 \mu \mathrm{g} / \mathrm{ml})$, glutamine $(0.1 \mathrm{mg} / \mathrm{ml})$ and pyruvate $(110 \mu \mathrm{g} / \mathrm{ml})$ at $38.8^{\circ} \mathrm{C}$ for $24 \mathrm{~h}$ in a humidified atmosphere containing $5 \%$ $\mathrm{CO}_{2}$ as described earlier (Donnay et al. 2004). $\mathrm{LiCl}_{2}$ (final concentration of $20 \mathrm{mM}$ if not specified) or BIO at $0.5-50 \mu \mathrm{M}$ was added in culture medium just before IVM. Oocytes were denuded by mechanical separation of CC either immediately after collection (immature oocytes at GV stage, $0 \mathrm{~h}$ of IVM) or after 3, 6, 10, 18 or 24 h of IVM. Oocytes were rinsed in PBS, frozen in liquid nitrogen and stored until RNA or protein extraction. In each experimental situation, 20-50 oocytes were analysed for their nuclear status. Meiotic status of oocytes at different times of maturation was established by lamin A/C immunofluorescence and chromatin labelling with Hoechst33342 or DAPI (Sigma, $1 \mu \mathrm{g} / \mathrm{ml}$ ), followed by microscopic observation. Corresponding CC were centrifuged and pellets were kept frozen at $-80{ }^{\circ} \mathrm{C}$ until use.

Granulosa cells from small follicles $(<6 \mathrm{~mm})$ were harvested by puncturing the follicles allowing expulsion of the cells. Cells were recovered by centrifugation, washed with fresh medium and counted in a haemocytometer. The culture medium used was McCoy 5A supplemented with $20 \mathrm{mM}$ HEPES, penicillin $(100 \mathrm{U} / \mathrm{ml})$, streptomycin $(100 \mathrm{mg} / \mathrm{l})$, L-glutamine $(3 \mathrm{mmol} / \mathrm{l})$, $0.1 \% \mathrm{BSA}, 0.1 \mu \mathrm{mol} / \mathrm{l}$ androstenedione, $5 \mathrm{mg} / \mathrm{l}$ transferrin, $20 \mu \mathrm{g} / \mathrm{l}$ selenium and 5\% FBS. The cells were initially cultured for $48 \mathrm{~h}$ and then serum starved for next $24 \mathrm{~h}$. Then cells were incubated in fresh culture medium with or without test reagents for the appropriate time. All granulosa cell cultures were performed under a water-saturated atmosphere of $95 \%$ air $/ 5 \%$ $\mathrm{CO}_{2}$ at $37^{\circ} \mathrm{C}$.

For RNA and protein extraction, we used samples of CC or entire COC, oocytes, primary culture of granulosa cells $\left(5 \times 10^{5}\right.$ cells per sample) and $1 \mathrm{~g}$ biopsy from hypothalamic region of adult cow brain. All samples were frozen and kept at $-80^{\circ} \mathrm{C}$ before experiments.

\section{Thymidine incorporation into granulosa cells}

Granulosa cells $\left(2 \times 10^{5}\right.$ viable cells/500 $\left.\mu \mathrm{l}\right)$ were cultured in 24-well dishes in McCoy 5A medium and 10\% FBS for $48 \mathrm{~h}$ and were then serum starved for $24 \mathrm{~h}$. Next, $1 \mu \mathrm{Ci} / \mu \mathrm{l}$ of $\left[{ }^{3} \mathrm{H}\right]-$ thymidine (Amersham Life Science) was added in the presence or absence of $\mathrm{LiCl}_{2}(20 \mathrm{mM})$ and/or FSH $\left(10^{-8} \mathrm{M}\right)$ and IGF-1 $\left(10^{-8} \mathrm{M}\right)$. Cultures were maintained at $37^{\circ} \mathrm{C}$ under $5 \% \mathrm{CO}_{2}$ in air. After $24 \mathrm{~h}$ of culture, excess of thymidine was removed by washing twice with PBS and the cells fixed with cold trichloroacetic acid 50\% for $15 \mathrm{~min}$ and lysed by addition of $0.5 \mathrm{~N} \mathrm{NaOH}$. The radioactivity was determined in scintillation fluid (Packard Bioscience) by counting in a $\beta$-photomultiplier.

\section{RNA and CDNA preparation and analysis}

\section{Total RNA preparation}

Total RNA was extracted using TriZol reagent as advised by the manufacturer (Invitrogen). To avoid contaminations with genomic DNA, total RNA preparations were treated by RQ1 DNAse (Promega) as described in the manufacturer's protocol.

\section{RT-PCR}

RT was performed on RNA amounts corresponding to 10 oocytes or on $1 \mu \mathrm{g}$ of total RNA from CC and brain. Complementary DNA was extended from oligo (dT)15 primers during $1 \mathrm{~h}$ at $37^{\circ} \mathrm{C}$ by mouse Moloney leukaemia virus reverse transcriptase (Invitrogen) as described in the user's manual. For RT-PCR analysis $1 \%$ of total RT reaction was used as a template. PCRs were performed using reagents from Interchim (Montluçon, France). In the negative control reaction, RNA amounts were directly subjected to PCR. Bovine GSK3A- and GSK3Bspecific primers at a final concentration $0.5 \mu \mathrm{M}$ (GSK3A: sense 5'-TGGCAGTCGACCGATAACACA and antisense 5'-GTTGGACGGTGTGAATCGACAT; GSK3B: sense 5'-TCCTTCAAATACCACAGCAGCC and antisense 5'-TTCCAATCGTGACCAGTGTTGC, Genbank accession numbers NM_001102192 and DT844404 respectively) were used to perform 35 cycles of PCR using the same cDNAs as templates. As a positive control of cDNA quality, 28 cycles of $\beta$-actin-specific PCR (primers $5^{\prime}$ GCGTGACATCAAGGAGAAGC and 5'-TGGAAGGTGGACAGGGAGGC) were performed on all cDNA samples. To confirm the specificity of amplified fragments, PCR products were cloned and sequenced.

\section{Protein analysis}

\section{Antibodies}

GSK-3 $\beta$ rabbit MAB, phospho-Ser ${ }^{21 / 9}$-GSK-3 $\alpha / \beta$, phosphop44/42 MAPK rabbit polyclonal antibody (MAPK3/MAPK1 phosphorylated at $\mathrm{Thr}^{202} / \mathrm{Tyr}^{204}$ respectively) and phospho-p38 (phospho-MAPK14) phosphorylated at $\mathrm{Thr}^{180} / \mathrm{Tyr}^{182}$ were purchased from Cell Signalling (Danvers, MA, USA). Total MAPK42 (MAPK1 or ERK2, C14) and p38 $\alpha$ (MAPK14, C20) rabbit polyclonal antibodies were purchased from Santa Cruz Biotechnology (Santa Cruz, CA, USA). $\alpha$-Tubulin (TUBA) MAB (clone DM1) was from Sigma. All primary antibodies used were raised against highly homologous human or mice (MAPK14) antigens and therefore cross-reacted with corresponding bovine proteins. HRP-conjugated rabbit anti-mouse, goat anti-rabbit and goat anti-mouse IgG antibodies were purchased from Lab Vision (Fremont, CA, USA). Lamin A/C MAB was from Ozyme (Saint Quentin Yvelines, France); Alexa Fluor488 goat anti-rabbit IgG and Alexa Fluor594 goat anti-mouse IgG were from Molecular Probes (Carlshad, CA, USA).

\section{Western immunoblotting}

Groups of a definite number of bovine oocytes, COC, cumulus or granulosa cells were lysed and then frozen-thawed in $20 \mu \mathrm{l}$ of Tris-saline-EGTA buffer ( $\mathrm{pH}$ 7.5) supplemented with $2 \mathrm{mM}$ sodium orthovanadate and $1 \mu \mathrm{l} / \mathrm{ml}$ of protease inhibitor cocktail (Sigma) then thawed-frozen three times by rapid incubation in liquid nitrogen followed by immersion in a warm water bath at $30{ }^{\circ} \mathrm{C}$. Before loading, concentrated reducing Laemmli buffer containing $80 \mathrm{mM}$ dithiothreitol at final concentration was added to all protein extracts and samples were boiled for $8 \mathrm{~min}$.

Proteins extracts were resolved on 10\% SDS-PAGE gels and transferred onto nitrocellulose membranes. Blots were blocked with 5\% dry milk in Tris-buffered saline/0.1\% Tween 20 for $1 \mathrm{~h}$ 
at room temperature and probed with various antibodies overnight at $4{ }^{\circ} \mathrm{C}$. Dilutions were $1 / 1000$ for all primary antibodies. After extensive washes in $1 \times \mathrm{TBS} /$ Tween $0.1 \%$, immunoreactivity was detected using the appropriate HRPconjugated secondary antibodies (diluted 1/5000, incubated $1 \mathrm{~h}$ at room temperature) and revealed by ECL Plus kit according to the manufacturer's instructions (Amersham Biosciences). Densitometry was performed by scanning the original radiographs and then analysing the bands with Scion Image software Beta 4.0.2 (Fuji PhotoFilm, Valhalla, NY, USA). At least three blots were analysed for each experimental condition. Quantified data was subjected to one-way ANOVA and/or Student's $t$-test at a minimum level of significance of $P<0.05$.

\section{Immunohistochemistry}

Ovarian biopsies (quarters of whole including cortex with follicles $<6 \mathrm{~mm}$ ) were fixed for $12 \mathrm{~h}$ in a solution containing $50 \%$ of saturated picric acid, $3.7 \%$ formaldehyde and $5 \%$ of acetic acid. After serial dehydration steps, the samples were embedded in paraffin and serially sectioned at a thickness of $7 \mu \mathrm{m}$. Sections were deparaffined, re-hydrated and microwaved for $5 \mathrm{~min}$ in antigen-unmasking solution (Vector Laboratories, Inc., AbCys, Paris, France) then left to cool at room temperature. After washing in a PBS bath for $5 \mathrm{~min}$, sections were immersed in a peroxidase-blocking reagent for $10 \mathrm{~min}$ at room temperature to quench endogenous peroxidase activity (Dako Cytomation; Dako, Ely, UK). After three 5-min washes in a PBS bath, sections were blocked with $5 \%$ goat serum in PBS for $20 \mathrm{~min}$ and then incubated overnight at $4{ }^{\circ} \mathrm{C}$ with $\mathrm{PBS} / 0.1 \%$ BSA containing GSK3 $\beta$ or phospho-GSK $3 \alpha / \beta$ antibody (dilution 1/100). After washing thrice for $10 \mathrm{~min}$, sections were incubated for $30 \mathrm{~min}$ at room temperature with a biotinylated goat anti-rabbit antibody. After serial washes, a 10 min staining was performed in a streptavidin peroxidase solution at room temperature as described in the kit manual (both Lab Vision Corporation). Immunoreactivity was revealed by incubation at room temperature with DAB. The slides were counterstained with haematoxylin, then dehydrated and mounted in Depex (Sigma). Negative controls were performed by replacing primary antibodies by rabbit $\lg$ diluted in PBS $/ 0.1 \%$ BSA at a final concentration of $2 \mu \mathrm{g} / \mathrm{ml}$. Slides were observed using the Axioplan Zeiss transmission microscope.

\section{Immunofluorescence of bovine oocytes}

Oocytes were fixed for $10 \mathrm{~min}$ in a solution containing $50 \%$ of saturated picric acid, $3.7 \%$ of methanol stabilisedformaldehyde (Interchim) and 5\% of acetic acid or in fresh

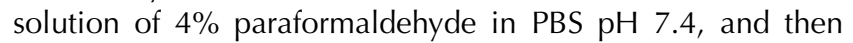
washed four times $15 \mathrm{~min}$ in PBS supplemented with $0.2 \%$ BSA and once $30 \mathrm{~min}$ in PBS/0.2\% BSA/0.1\% Triton. Blocking was performed in PBS supplemented with $2 \%$ BSA and $10 \%$ of goat inactivated serum during $2 \mathrm{~h}$. Overnight incubation with primary antibodies (diluted $1 / 100$ for phospho-Ser-GSK $3 \alpha / \beta$ and GSK3 $\beta, 1 / 200$ for lamin $\mathrm{A} / \mathrm{C}$ ) was performed at $4{ }^{\circ} \mathrm{C}$ with constant shaking. At least four 30 min washes in PBS/0.2\% BSA were done, and oocytes were incubated with corresponding secondary fluorochrome-conjugated antibody (diluted 1/200) for $2 \mathrm{~h}$ at room temperature. Five 20-min washes were then performed. Oocytes and embryos were put on slides and mounted with Mowiol supplemented with DABCO anti-fading and $1 \mu \mathrm{g} / \mu \mathrm{l}$ of DAPI (Sigma). Immunofluorescence was observed using Axioplan Zeiss fluorescent microscope supplied with appropriate filters.

\section{Progesterone and oestradiol RIA}

The concentration of progesterone in the culture medium of granulosa cells was measured after $48 \mathrm{~h}$ of culture by a RIA protocol as previously described (Tosca et al. 2005). Results were expressed as the amount of progesterone $(\mathrm{ng} / \mathrm{ml})$ secreted by granulosa primary cultured cells for $48 \mathrm{~h}$ per $100 \mu \mathrm{g}$ of protein. Progesterone secreted by groups of COC was measured by using $50 \mu \mathrm{l}$ of medium sampled at $0,1,3,6,10$ and $24 \mathrm{~h}$ after beginning of IVM using the same protocol. Results were expressed as the amount of progesterone $(\mathrm{ng} / \mathrm{ml})$ secreted by groups of $50 \mathrm{COC}$ for $24 \mathrm{~h}$. Statistical treatment was performed by ANOVA at a minimum level of significance of $P<0.05$.

\section{Declaration of interest}

The authors declare that there is no conflict of interest that could be perceived as prejudicing the impartiality of the research reported.

\section{Funding}

This work was a part of the OVOGENAE program, sponsored by ANR and APIS-GENE.

\section{References}

Acevedo N, Ding J \& Smith GD 2007 a Insulin signaling in mouse oocytes. Biology of Reproduction 77 872-879.

Acevedo N, Wang X, Dunn RL \& Smith GD $2007 b$ Glycogen synthase kinase-3 regulation of chromatin segregation and cytokinesis in mouse preimplantation embryos. Molecular Reproduction and Development 74 178-188.

Ali A, Hoeflich KP \& Woodgett JR 2001 Glycogen synthase kinase-3: properties, functions, and regulation. Chemical Reviews 101 2527-2540.

Baatout S, Jaussi R, Michaux A, Buset J, Schoonjans W \& Jacquet P 2007 Intracellular signal transduction in mouse oocytes and irradiated early embryos. In Vivo 21 587-592.

Baluch DP \& Capco DG 2008 GSK3 $\beta$ mediates acentromeric spindle stabilization by activated PKC zeta. Developmental Biology 317 46-58.

Bartolome J, Hernandez J, Landaeta A, Kelleman A, Sheerin P, Risco CA \& Archbal LF 2002 The effect of interval from day of administration of bovine somatotropin (bST) to synchronization of ovulation and timedinsemination on conception rate of dairy cows with and without ovarian cysts. Theriogenology 57 1293-1301.

Coenen K, Massicotte L \& Sirard MA 2004 Study of newly synthesized proteins during bovine oocyte maturation in vitro using image analysis of two-dimensional gel electrophoresis. Molecular Reproduction and Development 67 313-322.

Cohen P \& Frame S 2001 The renaissance of GSK3. Nature Reviews. Molecular Cell Biology 2 769-776.

Cohen P, Alessi DR \& Cross DA 1997 PDK1, one of the missing links in insulin signal transduction? FEBS Letters 410 3-10.

Donnay I, Faerge I, Grondahl C, Verhaeghe B, Sayoud H, Ponderato N, Galli C \& Lazzari G 2004 Effect of prematuration, meiosis activating sterol and enriched maturation medium on the nuclear maturation and competence to development of calf oocytes. Theriogenology 62 1093-1107. 
Fisher DL, Morin N \& Doree M 1999 A novel role for glycogen synthase kinase-3 in Xenopus development: maintenance of oocyte cell cycle arrest by a beta-catenin-independent mechanism. Development 126 567-576.

Frame S \& Cohen $\mathbf{P} 2001$ GSK3 takes centre stage more than 20 years after its discovery. Biochemical Journal 359 1-16.

Fumoto K, Lee PC, Saya H \& Kikuchi A 2008 AIP regulates stability of Aurora-A at early mitotic phase coordinately with GSK-3 $\beta$. Oncogene $\mathbf{2 7}$ 4478-4487.

Goodarzi MO, Antoine HJ, Pall M, Cui J, Guo X \& Azziz R 2007 Preliminary evidence of glycogen synthase kinase 3 beta as a genetic determinant of polycystic ovary syndrome. Fertility and Sterility 87 1473-1476.

Goodarzi MO, Jones MR, Chen YD \& Azziz R 2008 AKT2: first evidence of genetic association with polycystic ovary syndrome. Diabetes Care $\mathbf{3 1}$ 2284-2287.

Guo TB, Chan KC, Hakovirta H, Xiao Y, Toppari J, Mitchell AP \& Salameh WA 2003 Evidence for a role of glycogen synthase kinase-3 beta in rodent spermatogenesis. Journal of Andrology 24 332-342.

Harrell NB, Teachey MK, Gifford NJ \& Henriksen EJ 2007 Essential role of p38 MAPK for activation of skeletal muscle glucose transport by lithium. Archives of Physiology and Biochemistry 113 221-227.

Jope RS 2003 Lithium and GSK-3: one inhibitor, two inhibitory actions, multiple outcomes. Trends in Pharmacological Sciences 24 441-443.

Kaladchibachi SA, Doble B, Anthopoulos N, Woodgett JR \& Manoukian AS 2007 Glycogen synthase kinase 3, circadian rhythms, and bipolar disorder: a molecular link in the therapeutic action of lithium. Journal of Circadian Rhythms 53.

Lau KF, Miller CC, Anderton BH \& Shaw PC 1999 Expression analysis of glycogen synthase kinase-3 in human tissues. Journal of Peptide Research 54 85-91.

Lee SE, Kim JH \& Kim NH 2007 Inactivation of MAPK affects centrosome assembly, but not actin filament assembly, in mouse oocytes maturing in vitro. Molecular Reproduction and Development 74 904-911.

Li S, Maruo T, Ladines-Llave CA, Kondo H \& Mochizuki M 1994 Stagelimited expression of myc oncoprotein in the human ovary during follicular growth, regression and atresia. Endocrine Journal 41 83-92.

Liang CG, Su YQ, Fan HY, Schatten H \& Sun QY 2007 Mechanisms regulating oocyte meiotic resumption: roles of mitogen-activated protein kinase. Molecular Endocrinology 21 2037-2055.

Liu L, Rajareddy S, Reddy P, Jagarlamudi K, Du C, Shen Y, Guo Y, Boman K, Lundin E, Ottander U et al. 2007 Phosphorylation and inactivation of glycogen synthase kinase-3 by soluble kit ligand in mouse oocytes during early follicular development. Journal of Molecular Endocrinology $\mathbf{3 8}$ 137-146.

MacAulay K, Hajduch E, Blair AS, Coghlan MP, Smith SA \& Hundal HS 2003 Use of lithium and SB-415286 to explore the role of glycogen synthase kinase-3 in the regulation of glucose transport and glycogen synthase. European Journal of Biochemistry 18 3829-3838.

Meijer L, Flajolet M \& Greengard P 2004 Pharmacological inhibitors of glycogen synthase kinase 3. Trends in Pharmacological Sciences $\mathbf{2 5}$ 471-480.

Morrison DL, Yee A, Paddon HB, Vilimek D, Aebersold R \& Pelech SL 2000 Regulation of the meiosis-inhibited protein kinase, a p38MAPK isoform, during meiosis and following fertilization of Seastar oocytes. Journal of Biological Chemistry 275 34236-34244.

Parrott JA, Kim G \& Skinner MK 2000 Expression and action of kit ligand/stem cell factor in normal human and bovine ovarian surface epithelium and ovarian cancer. Biology of Reproduction 62 1600-1609.

Pascreau G, Delcros JG, Morin N, Prigent C \& Arlot-Bonnemains Y 2008 Aurora-A kinase Ser349 phosphorylation is required during Xenopus laevis oocyte maturation. Developmental Biology 317 523-530.

Pelech S, Jelinkova L, Susor A, Zhang H, Shi X, Pavlok A, Kubelka M \& Kovarova H 2008 Antibody microarray analyses of signal transduction protein expression and phosphorylation during porcine oocyte maturation. Journal of Proteome Research 7 2860-2871.

Perdiguero E, Pillaire MJ, Bodart JF, Hennersdorf F, Frodin M, Duesbery NS, Alonso G \& Nebreda AR 2003 Xp38gamma/SAPK3 promotes meiotic $\mathrm{G}(2) / \mathrm{M}$ transition in Xenopus oocytes and activates Cdc25C. EMBO Journal 22 5746-5756.
Salameh W, Helliwell JP, Han G, McPhaul L \& Khorram O 2006 Expression of endometrial glycogen synthase kinase-3beta protein throughout the menstrual cycle and its regulation by progesterone. Molecular Human Reproduction 12 543-549.

Sarkissian M, Mendez R \& Richter JD 2004 Progesterone and insulin stimulation of CPEB-dependent polyadenylation is regulated by Aurora $\mathrm{A}$ and glycogen synthase kinase-3. Genes and Development 18 48-61.

Sirard MA, Parrish JJ, Ware CB, Leibfried-Rutledge ML \& First NL 1988 The culture of bovine oocytes to obtain developmentally competent embryos. Biology of Reproduction 39 546-552.

Swain JE, Ding J, Wu J \& Smith GD 2008 Regulation of spindle and chromatin dynamics during early and late stages of oocyte maturation by aurora kinases. Molecular Human Reproduction 14 291-299.

Takenaka K, Moriguchi T \& Nishida E 1998 Activation of the protein kinase p38 in the spindle assembly checkpoint and mitotic arrest. Science 280 599-602.

Thornton TM, Pedraza-Alva G, Deng B, Wood CD, Aronshtam A, Clements JL, Sabio G, Davis RJ, Matthews DE, Doble B et al. 2008 Phosphorylation by 338 MAPK as an alternative pathway for GSK3 $\beta$ inactivation. Science 320 667-670.

Tomek W, Torner H \& Kanitz W 2002 Comparative analysis of protein synthesis, transcription and cytoplasmic polyadenylation of mRNA during maturation of bovine oocytes in vitro. Reproduction in Domestic Animals 37 86-91.

Tosca L, Froment P, Solnais P, Ferre P, Foufelle F \& Dupont J 2005 Adenosine $5^{\prime}$-monophosphate-activated protein kinase regulates progesterone secretion in rat granulosa cells. Endocrinology 146 4500-4513.

Tosca L, Uzbekova S, Chabrolle C \& Dupont J 2007 Possible role of 5'AMPactivated protein kinase in the metformin-mediated arrest of bovine oocytes at the germinal vesicle stage during in vitro maturation. Biology of Reproduction 77 452-465.

Uzbekova S, Arlot-Bonnemains Y, Dupont J, Dalbies-Tran R, Papillier P, Pennetier S, Thelie A, Perreau C, Mermillod P, Prigent C et al. 2008 Spatio-temporal expression patterns of aurora kinases $\mathrm{A}, \mathrm{B}$, and $\mathrm{C}$ and cytoplasmic polyadenylation-element-binding protein in bovine oocytes during meiotic maturation. Biology of Reproduction 78 218-233.

Vanholder T, Opsomer G \& de Kruif A 2006 Aetiology and pathogenesis of cystic ovarian follicles in dairy cattle: a review. Reproduction, Nutrition, Development 46 105-119.

Vigneault C, McGraw S, Massicotte L \& Sirard MA 2004 Transcription factor expression patterns in bovine in vitro-derived embryos prior to maternal-zygotic transition. Biology of Reproduction 70 1701-1709.

Vigneron C, Perreau C, Dalbies-Tran R, Joly C, Humblot P, Uzbekova S \& Mermillod P 2004a Protein synthesis and mRNA storage in cattle oocytes maintained under meiotic block by roscovitine inhibition of MPF activity. Molecular Reproduction and Development 69 457-465.

Vigneron C, Perreau C, Dupont J, Uzbekova S, Prigent C \& Mermillod P $2004 b$ Several signaling pathways are involved in the control of cattle oocyte maturation. Molecular Reproduction and Development 69 466-474.

Villa-Diaz LG \& Miyano T 2004 Activation of p38 MAPK during porcine oocyte maturation. Biology of Reproduction 71 691-696.

Wang X, Liu XT, Dunn R, Ohl DA \& Smith GD 2003 Glycogen synthase kinase-3 regulates mouse oocyte homologue segregation. Molecular Reproduction and Development 64 96-105.

Welsh GI, Miller CM, Loughlin AJ, Price NT \& Proud CG 1998 Regulation of eukaryotic initiation factor elF2B: glycogen synthase kinase-3 phosphorylates a conserved serine which undergoes dephosphorylation in response to insulin. FEBS Letters 421 125-130.

Woodgett JR 1990 Molecular cloning and expression of glycogen synthase kinase-3/factor A. EMBO Journal 9 2431-2438.

Yao HB, Shaw PC, Wong CC \& Wan DC 2002 Expression of glycogen synthase kinase-3 isoforms in mouse tissues and their transcription in the brain. Journal of Chemical Neuroanatomy 23 291-297.

Zarubin T \& Han J 2005 Activation and signaling of the p38 MAP kinase pathway. Cell Research 15 11-18.

Received 13 April 2009

First decision 21 May 2009

Accepted 21 May 2009 